\title{
NI CRÍTICA NI POST-CRÍTICA: POR UNA PEDAGOGía SIN ATRIBUTOS
}

\section{Neither critical nor post-critical: for a pedagogy without attributes}

\author{
Carlos Ernesto NOGUERA RAMÍREZ \\ Universidad Pedagógica Nacional. Colombia. \\ cnoguera@pedagogica.edu.co \\ bttps://orcid.org/0000-0001-9848-0724
}

Fecha de recepción: 15/02/2020

Fecha de aceptación: 08/04/2020

Fecha de publicación en línea: 01/07/2020

\section{RESUMEN}

El presente escrito constituye una respuesta al Manfiesto por una Pedagogía Post-crítca de Hodgson, Vlieghe y Zamojski. Parte del reconocimiento de puntos de coincidencia con los trabajos que algunos autores latinoamericanos han desarrollado al respecto, pero señala que el Manifiesto no consigue identificar el problema central de las pedagogías críticas. En esta dirección, el texto argumenta que el problema central de esas tendencias consiste en que su concepción de poder está circunscrita a lo que Foucault denominó las hipótesis bélica y represiva del poder. Tal forma de entender el poder, como confrontación o represión, hace que la educación se conciba como una acción política y, de esta manera, se invisibiliza su carácter fundamentalmente antropológico, o en términos de Sloterdijk, antropotécnico. Una de las consecuencias más significativas de este hecho es que la educación y la pedagogía viene sufriendo un proceso de politización frente al cual es necesaria y urgente una "crítica" de las "tendencias críticas" que se sustente en el horizonte conceptual de la pedagogía, y cuyo efecto sea su actualización y reconceptualización. Tal ejercicio permitiría producir nuevas herramientas que ayuden a comprender el funcionamiento de la educación como proyecto de la modernidad. La crítica de las tendencias críticas se plantea desde los conceptos de gobierno (Foucault) y antropotécnicas (Sloterdijk) que 
aplicados al análisis de la constitución del concepto moderno de educación llevan a proponer, siguiendo los análisis de Antelo y Serra, una pedagogía sin atributos, es decir, ni crítica ni post-crítica.

Palabras clave: pedagogía crítica; pedagogía post-crítica; antropotécnica; educación; gobierno.

\section{ABSTRACT}

The present document constitutes a response to the Manfiesto for a Post-critical Pedagogy of Hodgson, Vlieghe and Zamojski. Part of the recognition of points of coincidence with the works that some Latin American authors have developed in this regard but points out that the manifesto fails to identify the central problem of critical pedagogies. In this direction, the text argues that the central problem of these tendencies is that their conception of power is limited to what Foucault called the war and repressive hypotheses of power. Such a way of understanding power, as confrontation or repression, makes education conceived as a political action and, in this way, its fundamentally anthropological character (or in terms of Sloterdijk, anthropotechnical) is invisible. One of the most significant consequences of this fact is that education and pedagogy have been undergoing a process of politicization against which a "criticism" of the "critical tendencies» based on the conceptual horizon of pedagogy is necessary and urgent, and whose effect is its update and reconceptualization. Such an exercise would allow the production of new tools that help to understand the functioning of education as a modernity project. Criticism of the critical tendencies arises from the concepts of government (Foucault) and anthropotechnics (Sloterdijk) that applied to the analysis of the constitution of the modern concept of education lead to propose, following the analysis of Antelo and Serra, a pedagogy without attributes, that is, neither criticism nor post-criticism.

Key words: critical pedagogy; post-critical pedagogy; anthropotechnics; education; government.

En el medio educativo latinoamericano las corrientes críticas de la pedagogía gozan de un amplio reconocimiento y son seguidas por un número considerable de académicos y militantes (entre investigadores, profesores de enseñanza básica y media y estudiantes de instituciones de formación de maestros y facultades de educación). En este marco es de esperar que un manifiesto a favor de una postura post-crítica, formulado por investigadores europeos, no consiga despertar mayor entusiasmo y sea considerado, a lo sumo, como una cuestión típicamente europea. A pesar de este pronóstico, me he animado a escribir un texto para este número monográfico sobre el Manifiesto por una Pedagogía Post-crítica de los colegas 
Hodgson, Vlieghe y Zamojski $(2017)^{1}$ porque encuentro allí unos planteamientos que pueden movilizar una discusión necesaria en nuestro fragmentado y disperso campo académico.

Para entrar en materia quiero señalar que he encontrado en el Manifiesto puntos de coincidencia con los análisis que algunos investigadores hemos realizado durante las últimas décadas, en contravía de una tendencia mayoritaria en nuestro medio latinoamericano; no obstante, quiero también afirmar, como punto de partida, que a pesar de su intensión "crítica", considero que los colegas europeos no consiguen acertar en el núcleo problemático de los enfoques críticos sobre la educación (y la pedagogía). Me parece que el enfoque de los autores del Manifiesto no da en el blanco y, de esa manera, soslayan un asunto crucial para la pedagogía y la educación en el mundo contemporáneo. Sobre la base de estas dos premisas y como una contribución al espíritu del Manifiesto, me propongo con este texto hacer algunas precisiones a los principios propuestos en la perspectiva de iniciar una conversación que nos abra nuevos horizontes para pensar lo que acontece hoy en el mundo de la educación y la pedagogía.

\section{1. ¿MANiFiESTO O DECLARACIÓN?}

Una primera precisión tiene que ver con el carácter del texto, es decir, con su condición de manifiesto. En este punto coincido plenamente con Lewis (2017) en su respuesta al texto de Hodgson, Vlieghe y Zamojski (2017) cuando afirma que, en realidad, no se trata de un manifiesto sino más bien de una declaración. No es este un asunto menor, como lo muestra Lewis, pues la forma de escritura tiene una íntima relación con su contenido. El manifiesto es un género típicamente crítico, baste recordar el más famoso de ellos, el de Marx y Engels. Pero Lewis nos recuerda, también, los manifiestos de Giroux (2018) y McLaren (2009) y, además, nos señala sus características fundamentales: se trata de un emprendimiento colectivo que articula no simplemente una serie de creencias personales sino, por el contrario, el punto de vista de una clase; es polémico, es transgresor de la ley, de lo establecido y, por último, es profético, pues está orientado hacia el futuro, hacia la transformación de un estado de cosas.

En este sentido, si bien el texto de Hodgson, Vlieghe y Zamojski (2017) parece corresponder, en primera instancia, a estas características, definitivamente su orientación, como señala uno de sus principios, no es hacia un futuro, no es profético, por el contrario, parte de reconocer una esperanza en el presente en la medida en que afirma que hay cosas del presente dignas de cuidar y transmitir. Esta esperanza se opone, a su vez, al carácter salvacionista y esclarecido que se sustenta en la

1. La versión española que se cita en este artículo forma parte del monográfico «Una respuesta iberoamericana al 'Manifiesto por una pedagogía post-crítica'»: http://dx.doi.org/10.14201/teri.22862 
acción de los intelectuales críticos (y que forma parte de cualquier manifiesto) de desvelar cuestiones que han sido ocultadas por mezquinos intereses y que no son percibidas por las inteligencias promedio de la población. Por el contrario, lo que proponen Hodgson, Vlieghe y Zamojski (2017) es proteger y cuidar, una especie de postura conservadora soportada en la acción de retomar la pregunta por el sentido de conceptos como educación, crianza, escuela, estudio, pensamiento y práctica.

\section{2. ¿Pedagogía crítica o pedagogías críticas?}

Una segunda precisión, también de carácter general, tiene que ver con lo que los colegas europeos denominan "pedagogía crítica": ¿existe una pedagogía crítica? A pesar de las diferencias susceptibles de identificar en las distintas corrientes críticas ¿podríamos agruparlas en una única tendencia crítica general?, ¿no sería más adecuado hablar de pedagogías críticas? Este punto es particularmente importante, como lo muestra el caso de Peter McLaren: ¿se podría aplicar la afirmación que Hodgson, Vlieghe y Zamojski sostienen a propósito de la centralidad de las políticas de identidad en su teoría de la educación? En otros términos: ¿podría el enfoque de McLaren ser clasificado como posestructuralista y posmoderno? Miremos lo que afirma este autor en una entrevista recientemente publicada (2012, p. 17):

Ha habido tiempos en los que he coqueteado con la teoría posmoderna, con la apostasía en boga del bandolerismo posestructuralista, o el bandidaje deconstruccionista — hasta el punto en que he sido identificado como el primero en introducir el término posmodernismo en el léxico de la crítica educativa (una afirmación dudosa, pero no obstante, hecha sobre mi trabajo)—, pero he encontrado que existen limitaciones insuperables al trabajo, para no mencionar una creciente confederación de sicofantes académicos que en estos días parecen sobrepoblar los estudios críticos norteamericanos y europeos.

De acuerdo con lo anterior, el enfoque de McLaren no correspondería a la "pedagogía crítica» que los autores del Manifiesto interrogan, por lo cual, deberíamos entender que este se refiere, en particular, a las corrientes posmodernas o posestructuralistas. Además del caso McLaren, muchas otras corrientes se localizan fuera de la línea posmoderna y posestructuralista, como lo muestra el Manifiesto latinoamericano de pedagogía crítica y educación popular (2015) centrado en los conceptos de lucha de clases y mercantilización. Sin embargo, ello no significa que sea imposible hablar de la pedagogía crítica como un conjunto de corrientes cuyo núcleo común es, justamente, su perspectiva crítica, solo que es necesario reconocer que esa crítica se realiza desde distintas perspectivas y ahí es donde se encuentran las diferentes posiciones. 


\section{El NúCleo PROBLEMÁtico de las PEDAGogías CRíticas}

Ahora bien, si la crítica se realiza desde distintos ángulos, por ejemplo, marxistas y neomarxistas (que implican diferencias fundamentales) ¿̨cómo se podría aun hablar de una tendencia crítica general? ¿en qué consistiría el núcleo de la crítica? Me parece que lo común a las corrientes críticas, no es la comprensión de la educación en términos de políticas de identidad, como lo señalan los autores del Manifiesto (esta es una característica de algunas corrientes); la característica central y cuestionable de las tendencias críticas es la manera de entender el poder y su relación con la educación, lo que deriva, a su vez, en una manera particular de comprender lo que ha sido la educación calificada como «moderna» o "tradicional». Y ahí es donde considero pertinente pensar en una mirada crítica sobre las pedagogías críticas, perspectiva que no puede confundirse con una pedagogía post-crítica, como explicaré más adelante.

¿En qué consiste esa manera de entender el poder y su relación con la educación que caracterizaría a las distintas corrientes críticas en la pedagogía? Para estas corrientes el poder es guerra, confrontación o represión, coerción; se trata de entender el poder desde una hipótesis bélica o «hipótesis Nietzsche» o desde una hipótesis represiva o «hipótesis Reich» (Foucault, 2001a). Estas hipótesis son predominantes en la comprensión de las ciencias sociales y la filosofía política y frente a ellas Foucault exploró las condiciones arqueológicas y genealógicas que las hicieron predominantes, en la perspectiva de abandonarlas para comprender mejor las nuevas formas de ejercicio del poder que, por la década de 1970, se extendían por el espacio social como parte de lo que llamó nuevas «luchas transversales» del poder (Foucault, 2001b). Lo anterior no significa una negación ni de la opresión ni de la confrontación social: sería ingenuo, idealista o perverso negar la existencia de la explotación y de la dominación, así como de la confrontación social; sin embargo, y esa es la apuesta de Foucault, se trata de crear nuevas herramientas para comprender las nuevas formas como opera el poder, más sutiles, menos evidentes y, sobre todo, ligadas íntimamente al ejercicio individual de la libertad².

2. Sobre este punto, es su curso Nacimiento de la biopolítica Foucault (2008), refiriéndose a la gubernamentalidad liberal, es decir, a la forma liberal de gobierno, señala: "Si empleo el término 'liberal' es ante todo porque esta práctica gubernamental (...) no se conforma con respetar tal o cual libertad, garantizar tal o cual libertad. Más profundamente, es consumidora de libertad. Y lo es en la medida en que solo puede funcionar si hay efectivamente una serie de libertades: libertad de mercado, libertad del vendedor y el comprador, libre ejercicio del derecho de propiedad, libertad de discusión, eventualmente, libertad de expresión, etc. Por lo tanto, la nueva razón gubernamental tiene necesidad de libertad, el nuevo arte gubernamental consume libertad. Consume libertad: es decir, que está obligado a producirla» (pp. 83-84). Y más adelante continúa: "El liberalismo no es lo que acepta la libertad, es lo que se propone fabricarla a cada momento, suscitarla, producirla con, desde luego, [todo el conjunto] de coacciones, problemas de costo que plantea esa fabricación” (p. 85). 
Ahora bien, si el poder es confrontación o represión, ¿qué tipo de relaciones se desprenden desde estas hipótesis sobre la educación? A mi modo de ver, en la respuesta a este interrogante está la clave que justificaría la pertinencia de un «manifiesto» a favor de la pedagogía. Es decir, debido a la manera como las corrientes críticas entienden la relación entre poder y educación, tiene sentido hoy reclamar (y proclamar) una "crítica» de las «tendencias críticas» que permita pensar más allá de las hipótesis bélica y represiva del poder con el ánimo de comprender mejor lo que significó la educación moderna y su estrecha relación con las formas disciplinarias, liberales y neoliberales de gobernamiento ${ }^{3}$. Retomando la pregunta anterior, podría decirse que para las corrientes críticas la educación (tradicional) es un acto de poder (o coerción o represión) que unos (padres, profesores, adultos) ejercen sobre otros (infantes, estudiantes, jóvenes u otros adultos) con el propósito de conservar y reproducir un statu quo. Por el contrario, la educación crítica renuncia a considerarse como un acto de poder (o coerción o represión) para comprenderse o como una acción que unos (padres, profesores, adultos) realizan juntamente con otros (infantes, estudiantes, jóvenes u otros adultos) con el propósito de conseguir, a través de la toma de conciencia de las condiciones de opresión y explotación, la humanización y, por tanto, la correspondiente liberación.

En otras palabras, las corrientes críticas convirtieron la educación en un asunto fundamentalmente político, generando una politización de la educación (y de la pedagogía) que se expresa en dos vías: por una parte, para estas perspectivas la educación tradicional (o burguesa) ideologiza, reprime y oprime; por otra parte - y en contrapartida - la educación crítica concientiza, emancipa, libera. He ahí el núcleo problemático de las tendencias críticas. Contraria a esta perspectiva, podría retomarse la tradición del pensamiento pedagógico en una clave no política sino pedagógica. Desde mi perspectiva, es eso, justamente, lo que hizo Freire (2005) con la pedagogía del oprimido cuando consideró que la revolución tenía un carácter eminentemente pedagógico, lo que significa que la acción revolucionaria debe ser, en lo fundamental, una acción educativa. Con esa afirmación la pedagogía del oprimido, antes que una politización de la pedagogía es, claramente, una pedagogización de la política.

Hay otra cuestión bastante problemática que se deriva de esa politización de la educación introducida por las corrientes críticas debido a su manera de entender el poder: se trata de la ocultación o negación del carácter fundamentalmente antropológico (o antropotécnico ${ }^{4}$ ) de la educación. Según lo reconoce Fiori (2005,

3. Utilizo este término para no confundirlo con "gobierno" y por su proximidad con la noción de gubernamentalidad acuñada por Foucault en su curso Seguridad, Territorio, Población (2006).

4. En su polémica conferencia de 1999 titulada "Reglas para el parque humano", Peter Sloterdijk (2011) acuña el término antropotécnicas para referirse al conjunto de instrumentos y prácticas que los hombres utilizan para su crianza, domesticación y producción. Una década después (2009), en su libro titulado en español "Has de cambiar tu vida" (publicado en 2012), el filósofo alemán avanza en 
p. 17) en su prólogo a la pedagogía del oprimido: «la pedagogía es antropología", pues implica la producción del hombre o, en términos del propio Freire, su humanización. La educación se justifica por el carácter abierto e inacabado del ser humano: «Los hombres, diferentes de los otros animales, que son solo inacabado mas no históricos, se saben inacabados, inconclusos (...) Así se encuentra la raíz de la educación misma, como manifestación exclusivamente humana» (p. 97). Este aspecto fue claramente señalado por los humanistas del siglo XVI, en particular por Erasmo quien consideraba que la educación no era un ornato para embellecer al hombre, sino una necesidad propia del ser humano. No basta nacer para ser humano; la forma no constituye al hombre, es preciso que el recién nacido sea educado, pues de lo contrario crecerá como un animal. Si se trata de alcanzar la humanidad es necesario formar al hombre (Erasmo, 1956).

Llama la atención cómo hasta el propio Freire en su crítica a la educación tradicional — que califica de «educación bancaria»— confunda acciones destinadas a mantener y prolongar el sometimiento, la ignorancia, la simple obediencia, la naturalización de la desigualdad con una acción educativa. La idea freireana del "Ser más» que acompaña la educación liberadora, encuentra su emergencia, por ejemplo, en la didáctica comeniana que apunta a hacer del animal humano «algo más», una criatura racional y autónoma por efecto de la erudición, la virtud y la piedad. En este sentido, toda educación es humanizante y apunta a un "ser más»; por eso mismo, cualquier acción deshumanizante no puede ser considerada como una acción educativa. En términos genealógicos diríamos que la educación es una antropotécnica para el mejoramiento del género humano (Sloterdijk, 2012) y no una técnica de dominación. Desde luego, existieron y continúan existiendo ideales y técnicas de subyugación, dominación, represión, pero ninguna de ellas puede ser considerada educativa.

\section{4. ¿Post-CRítica O CRítica Radical a las «PEDAgogías CRíticas»?}

Hodgson, Vlieghe y Zamojski (2017) afirman que su Manifiesto en ningún caso constituye una postura anti-crítica, pues fue gracias al poderoso aparato crítico desarrollado a lo largo del siglo XX — dicen ellos- que pudimos conocer las características del statu quo en el que estamos inmersos. Por esa razón considero que calificar de post-crítica a una pedagogía que busca cuestionar los fundamentos de las corrientes críticas puede generar confusión acerca del objetivo que se propone.

\footnotetext{
la elaboración del concepto antropotécnicas al referirlo a los procedimientos de ejercitación tanto de tipo corporal como mental con los que los hombres de las diversas culturas «han intentado optimizar su estado inmunológico frente a los vagos riesgos de la vida y las agudas certezas de la muerte» (Sloterdijk, 2012, p. 24). Sobre la base de esta definición, y a través de sus cerca de 600 páginas, Sloterdijk emprende la construcción de una "teoría general del ejercicio» apoyándose para ello en la idea nietzscheana del humano como un ser ejercitante y de la Tierra como el astro ascético.
} 
En ese mismo sentido, rechazar el acto de "desenmascaramiento o develamiento" y oponerlo a la idea de "protección y cuidado" que nos debe llevar a preguntarnos de nuevo "por lo que son la educación, la crianza, la escuela, el estudio, el pensamiento y la práctica" parece una postura opuesta a la crítica. Justamente, es a través de la crítica que podemos preguntarnos de nuevo por el sentido de esos conceptos y de esas prácticas sobre las cuales las corrientes críticas instauraron el velo del poder impidiendo, de esa manera, percibir su carácter antropotécnico.

En ese mismo sentido, vale la pena recordar la afirmación de Tomaz Tadeu da Silva (1999) según la cual el post-modernismo señala el fin de la pedagogía crítica y el comienzo de la pedagogía post-crítica en la medida en que acaba con cualquier vanguardismo, certeza y pretensión de emancipación. La postura de Silva ha tenido una amplia difusión en el ámbito latinoamericano, de tal manera que un Manifiesto a favor de una "pedagogía post-crítica" se asocia, fácilmente, con una perspectiva post-moderna, generando confusión en los propósitos que persigue.

Como consecuencia de las anteriores apreciaciones, ratifico la inconveniencia de hablar de una pedagogía post-crítica y, por el contrario, enfatizo la idea de no abandonar la crítica o de dejarla solo en manos de las tendencias críticas. Recuperar la crítica para la pedagogía significa, en primer lugar, una reconsideración de la educación como un asunto antropotécnico y, por tanto, un distanciamiento de su vinculación con formas de poder opresivas o represivas, es decir, un distanciamiento del núcleo temático propuesto por las llamadas pedagogías críticas. En últimas, se trata de reconocer que la educación - aunque tenga implicaciones políticas- no tiene un carácter político en sí misma. Más allá de la forma como las tendencias críticas entienden el poder, la postura crítica que propongo reconoce, para el caso de la educación, el poder como una cuestión de gobierno (Foucault, 2006), es decir, como una acción de conducción de la conducta propia y de los otros. Esto significa que, si bien la educación es una forma de ejercicio de poder, no se trata ni de dominación ni represión, sino de conducción, algo así como «(...) un gobierno no violento de los hombres que, mediante el [auto]control de su vida afectiva y moral, mediante el conocimiento de los secretos de su corazón y mediante el empleo de una pedagogía finamente individualizada, procura conducirlos a la perfección» (Senellart, 2006, p. 29). El gobierno tiene, entonces, una procedencia filosófica (judeocristiana) y no política y la educación moderna, tal como se constituyó desde el Renacimiento, constituye una de las principales formas de gobierno de la población y de los individuos. Entendida desde esta perspectiva, cobran sentido varios elementos relacionados con los principios anunciados por Hodgson, Vlieghe y Zamojski en su Manifiesto.

En primer lugar, la apuesta de una educación —más allá de las diferencias y las particularidades que nos dividen- por un espacio de lo común, por una comunalidad. Algunas corrientes críticas de la pedagogía se han concentrado en la defensa de una educación que respete las particularidades de clase, género y etnia 
bajo una idea de poder como confrontación o represión y, por esa vía, lo común se diluye en las identidades. Aquí valdría la pena recordar a Freire (2005) para quien la pedagogía del oprimido no es una pedagogía para una clase, no es exclusivamente para los oprimidos, sino también para los opresores, pues en cuanto pedagogía liberadora, su horizonte es la humanidad y la humanización, de ahí su sintonía con el horizonte conceptual de la pedagogía moderna.

En segundo lugar, la crítica a las instituciones sociales y su disfuncionalidad, planteada desde las miradas tradicionales del poder, ha politizado las relaciones entre adultos e infantes y, en general, entre profesores y estudiantes, de tal modo que prácticas como la crianza, la educación son consideradas actos de opresión o represión y actividades como el estudio y el pensamiento, acciones de carácter ideológico. De ahí que, como señalan Hodgson, Vlieghe y Zamojski en el Manifiesto (2017), sea necesario:

(...) crear un espacio de pensamiento que permita que la práctica tenga lugar de nuevo. Esto significa (re)establecer nuestra relación con las palabras, abriéndolas a cuestionamientos, y prestar atención filosófica a aquellos aspectos devaluados de nuestras formas de vida, y, de ahí — de acuerdo con una normatividad de principios- defender estos acontecimientos como autotélicos y no funcionalistas, simplemente porque merecen la pena ser cuidados.

En tercer lugar, abandonar el papel del educador como aquel que desvela o desenmascara el poder (la opresión y la represión) no significa, necesariamente, aceptar o justificar el actual estado de cosas. Una crítica a las tendencias críticas, pasa por considerar al educador como alguien que tiene la capacidad de reconocer el valor de algunas de las cosas que hacemos (y la manera como las hacemos), alguien que sabe identificar aquello que merece ser preservado y, por tanto, aquello que es digno de ser transmitido. Nuevamente, vale la pena recordar a Freire cuando señalaba que el paso de la consciencia ingenua a la consciencia crítica no significaba un rechazo de la ciencia y del conocimiento, así como la liberación no reñía con la disciplina y el estudio (Freire, 2018).

En cuarto lugar, el distanciamiento de las tendencias críticas marxistas o la crítica de esas posiciones, en los términos en que lo he venido planteando, parte de la confirmación de que (Hodgson, Vlieghe y Zamojski, 2017):

Ha llegado el momento de reconocer y afirmar que hay cosas buenas en el mundo que merecen ser preservadas. Ha llegado el momento de que al desenmascaramiento del mundo le siga un reconocimiento esperanzado del mundo. Ha llegado el momento de situar lo que de bueno hay en el mundo - aquello que se encuentra amenazado y que deseamos preservar - en el centro de nuestra atención y de abrir un espacio conceptual en el que podamos aceptar nuestra responsabilidad sobre todo ello, frente a y a pesar de la opresión y la melancolía silenciosa. 
El anterior enunciado del Manifiesto es particularmente importante, pues varias tendencias críticas con su belicismo, suspicacia y prevención han caído en el resentimiento, el rechazo y el odio al mundo. Reconocer que hay cosas que merecen ser preservadas y transmitidas no significa querer que el mundo siga igual, no significa rechazar la posibilidad del cambio. La educación, en su sentido moderno, implicaba perfeccionamiento, mejoramiento o, como diría Freire, un «ser más» que parte, no del odio al mundo sino de "un esperanzador reconocimiento del mundo", de una "pedagogía de la esperanza".

¿Cómo entender, entonces, una postura crítica frente a las tendencias críticas marxistas? De lo que se trata es de retomar la crítica en su sentido moderno, lo cual, siguiendo a Foucault (2007), significa retomar la tradición kantiana de la crítica, es decir, poner a funcionar una ontología histórica del presente 5 .

Para efectos de procedimiento, Foucault identifica una dimensión arqueológica del análisis, centrada en las positividades discursivas, en los efectos de conocimientos aceptables en un momento dado y una dimensión genealógica cuyo propósito es «restituir las condiciones de aparición de una singularidad a partir de múltiples elementos determinantes, de los que no aparece como el producto sino como el efecto» (2007, p. 31). De esta manera, la arqueología y la genealogía como herramientas de una actitud crítica no tienen como propósito desvelar los errores, la ilusión, las oscuras intenciones detrás del saber ni poner en evidencia el poder o las formas de dominación que se ocultan tras el saber: como actitud crítica vinculada a la tradición de la modernidad, de la Ilustración, la actitud crítica es más bien una decisión de salir de la minoría de edad, de hacerse una idea justa de nuestras verdades, de hacer uso de ciertas herramientas para determinar los límites de lo sabido, de lo conocido, de lo pensado, en últimas, una opción por no ser gobernado de cierta forma, en vista de ciertos propósitos y por medio de ciertos procedimientos que llegaron a convertirse en legítimos en el campo de las llamadas ciencias sociales y humanas.

5. «La ontología crítica de nosotros mismos no hay que considerarla, ciertamente, como una teoría, como una doctrina, ni siquiera un cuerpo permanente de saber que se acumula; hay que concebirla como un ethos, una vida filosófica en la que la crítica de lo que somos es a la vez análisis histórico de los límites que nos son impuestos y prueba de su posible transgresión.

Esta actitud filosófica debe traducirse en un trabajo de investigaciones diversas: éstas tienen su coherencia metodológica en el estudio a la vez arqueológico y genealógico de prácticas consideradas simultáneamente como tipos tecnológicos de racionalidad y como juegos estratégicos de libertades; tienen su coherencia teórica en la definición de las formas históricamente singulares bajo las cuales has sido problematizadas las generalidades de nuestra relación con las cosas, con los otros y con nosotros mismos. Tienen su coherencia práctica en el cuidado con que someten las reflexión histórico-crítica a la prueba de las prácticas concretas. No sé si hoy en día es necesario decir que el trabajo crítico implica todavía la fe en las Luces; necesita, siempre, creo yo, un trabajo sobre nosotros mismos, es decir, una labor paciente que dé forma a la impaciencia de la libertad» (Foucault, 2007, p. 97). 
CARLOS ERNESTO NOGUERA RAMÍREZ

\section{Por una pedagogía sin atributos}

Ni pedagogías críticas ni pedagogía post-crítica: en la línea de los análisis de los colegas argentinos Antelo y Serra (2013), nuestro reto debería ser una "pedagogía sin atributos». Por la historia de la educación, sabemos que la pedagogía es un saber moderno y, en ese sentido, las tendencias críticas nacen como una oposición o denuncia a la pedagogía moderna. Una pedagogía post-crítica sería una pedagogía que viene después de las pedagogías críticas, una pedagogía que las supera o abandona, de ahí la afirmación de Silva (1999) sobre el carácter post-moderno de tal pretensión. En la perspectiva de fortalecer la apuesta de Hodgson, Vlieghe y Zamojski (2017), propongo, entonces, utilizar la crítica como una herramienta para retomar el horizonte conceptual de la pedagogía (Zuluaga, 1999), actualizarlo y reconceptualizarlo de tal manera que se devele o se haga visible el carácter antropotécnico de la educación: y ello solo será posible si la concebimos, no como un poder represivo u opresivo, sino como una acción de gobierno, de gobernamiento de sí mismo y de los otros o como la antropotécnica fundamental creada en la modernidad y que, a su vez, creó la propia modernidad. Por tal motivo - y siguiendo los análisis de Antelo y Serra (2013) — el pensamiento crítico [el enfoque auto-denominado crítico] necesita ser interrogado, pues:

Si revisamos cómo las teorías críticas han dialogado con la constitución del campo de la educación en las últimas décadas, nos encontramos con un panorama más complejo. Por momentos, pareciera que las críticas a los sistemas educativos propios de los estados nacionales (su forma piramidal, su base capitalista en el ideal de ciudadanía que sostienen, su matriz homogeneizante, etc.) hasta habilitaron lo que sucedió en la década de los noventa: un estado mutante, desdibujado, ambiguo, que en nombre de la participación, la autonomía, la necesidad de dar la palabra, los proyectos institucionales, el reconocimiento de los particularismos y las identidades plurales, dejó de disciplinar, formatear, instituir, ordenar, etc. A su vez, el sujeto crítico, participativo y autónomo se convierte en una especie de empresario de sí, obligado a hacerse cargo del imperativo del propio éxito o la responsabilidad del propio fracaso (p. 68).

De alguna manera, las pedagogías críticas, al oponerse a la pedagogía tradicional, terminaron haciéndole el juego a las perspectivas neoliberales, por lo que habría que contradecir a Silva (1999) afirmando que no son la pedagogía tradicional y la pedagogía crítica quienes acabaron convergiendo en una genealogía común, sino que fueron las pedagogías críticas y aquellas que podríamos catalogar como neoliberales, las que, finalmente - unidas por su crítica a la pedagogía modernaterminaron confluyendo en una misma línea genealógica, tal como lo insinúan Antelo y Serra (2013). Una pedagogía sin atributos, por el contrario, se opone a unas y a otras mediante la creación de un espacio para pensar de nuevo los conceptos, las prácticas y las técnicas educativas.

Una pedagogía sin atributos estaría íntimamente ligada al proyecto de formación de humanos y humanidad que se llamó «modernidad» (Noguera, 2012). Ese proyecto 
consistió en la aplicación a una escala nunca antes vista de técnicas (antiguas y nuevas) para la producción en masa de seres humanos. Y aquí producción quiere decir formación, conformación, a partir de técnicas diversas bajo el principio de que el ser humano no nace, sino que se hace, no tiene un destino fijado por naturaleza, sino que debe construirlo a través de una segunda naturaleza que es la educación. Ahora bien, tal proyecto no estuvo exento de violencia, de explotación, de represión, pero esas prácticas no pueden confundirse con la educación. Sloterdijk (2012) nos ayuda a comprender lo que ello significó:

Solo se podrá hacer totalmente justicia a la Edad Moderna si se la relaciona con un cambio mental, moral y técnico hasta ahora nunca adecuadamente expuesto: la existencia de los tiempos modernos presenta rasgos de un ejercicio global de fitness donde lo que antes hemos llamado la «diferenciación ética», el llamado intensivo a la elevación de la vida — solo emprendida en la época pre-moderna por una minoría - se transformaría en un imperativo metanoético dirigido a todos y al que se dan múltiples respuestas. Sus mediadores serían, en primera línea, el Estado moderno y la escuela adecuada a él, con el apoyo enérgico al principio de los ministros de todas las confesiones (p. 427).

De esta manera, con ayuda de conceptos de Foucault (2001a, 2006, 2008) y Sloterdijk $(2011,2012)$, podemos declarar la urgencia de una crítica a las tendencias críticas (y ya no de un Manifiesto a favor de una postura post-crítica) que nos permita pensar de nuevo, que nos permita, para ello, construir nuevas herramientas para comprender cómo operan hoy las prácticas educativas. Para ello, nuevamente, las palabras de Antelo y Serra (2013) vienen en nuestro auxilio:

Hoy, por pedagogías críticas reconocemos a un grupo de pensadores y a un conjunto de problemas que tienen en común, entre otros rasgos, ligar la educación a la emancipación de los sujetos. Evidentemente, mucho de ese pensamiento resultó novedoso en los tiempos en que fue formulado, y en ese sentido, puede haber introducido todo un andamiaje procedimental como pensamiento que provocó efectos desestabilizantes en las certezas que la pedagogía daba por buena. Pero, ¿cumple en el presente el mismo objetivo? ¿Funciona el procedimiento crítico como posibilitador de una reflexión que resulta novedosa en el tratamiento de los problemas de la educación? La sensación es más bien la contraria: las herramientas conceptuales que tenemos parecen haber perdido la capacidad de inquietar, movilizar, desestabilizar, interrogar, producir, inventar... más bien parecen sedimentadas, solidificadas, por lo que habrían perdido su capacidad de intervenir (p. 73).

Pensar de nuevo es, pues, realizar un nuevo balance de las tradiciones pedagógicas modernas diferenciando técnicas y prácticas según sus posibilidades de formar seres humanos, es decir, según su carácter antropotécnico. Ello implica, además, revisar la relación que las pedagogías críticas establecieron entre poder y educación para reconsiderar la educación como una cuestión de gobierno, de gobernamiento, de conducción, No podemos renunciar al gobierno, no podemos eludir el gobierno, lo que podemos es gobernarnos de otra manera, por otros medios, para otros fines. 
CARLOS ERNESTO NOGUERA RAMÍREZ

NI CRÍTICA NI POST-CRÍTICA: POR UNA PEDAGOGÍA SIN ATRIBUTOS

Aquí deberíamos recordar al viejo Kant (2002) cuando señalaba en su curso de pedagogía que gobernar y educar son las dos artes más difíciles.

\section{REFERENCIAS BIBLIOGRÁFICAS}

Arendt, H. (2003). La crisis de la educación. En, Entre pasado y futuro. Ocho ejercicios sobre la reflexión política (pp. 169-301). Trad. Ana Luisa Poljak Zorzut. Barcelona: Península.

Antelo, R. y Serra, M. (2013). ¿Una pedagogía sin atributos? Revista Interuniversitaria de Formación del Profesorado, 27(2), 65-75.

Comenio, J. (1984). Didáctica magna. México: Porrúa.

Compayrè, G. (1897). Cours de pédagogie théorique et practique. Paris: Librairie Hachette.

Corominas, J. (1954). Diccionario crítico etimológico de la lengua castellana. Madrid: Gredos.

Erasmo. (1956). De cómo los niños precozmente y desde su nacimiento deben ser iniciados en la virtud y en las buenas letras. En, Obras escogidas (pp. 919-962). Madrid: Aguilar.

Fiori, E. M. (2005). Aprender a decir su palabra. El método de alfabetización del profesor Paulo Freire. En P. Freire, Pedagogía del oprimido (pp. 11-27). México: Siglo XXI.

Foucault, M. (2001a). Defender la sociedad. Buenos Aires: Fondo de Cultura Económica.

Foucault, M. (2001b). El sujeto y el poder. En H. Dreyfus y P. Rabinow, Michel Foucault: más allá de la hermenéutica y el estructuralismo (pp. 241-260). Buenos Aires: Nueva Visión.

Foucault, M. (2006). Seguridad, territorio, población. Buenos Aires: Fondo de Cultura Económica.

Foucault, M. (2007). Sobre la Ilustración. Madrid: Tecnos.

Foucault, M. (2008). Nacimiento de la biopolítica. Buenos Aires: Fondo de Cultura Económica.

Freire, P. (2005). Pedagogía del oprimido. México: Siglo XXI.

Freire, P. (2018). Pedagogía de la autonomía. México: Siglo XXI.

Giroux, H. (2018). Manifiesto por una pedagogía crítica. En R. Aparici, C. Escaño y D. García Marín (Coords.), La otra educación. Pedagogías críticas para el siglo XXI (pp. 307-321). Madrid: UNED.

Hodgson, N., Vlieghe, J. y Zamojski, P. (2017). Manifesto for a Post-critical Pedagogy. London: Punctum Books. Recuperado de: https://punctumbooks.com/titles/manifesto-for-a-postcritical-pedagogy/ (Consultado el 08/04/2020).

Kant, I. (2002). Pedagogía. Madrid: Akal.

Lewis, T. (2017). A response to the "Manifesto for A Post-Critical Pedagogy». En N. Hodgson, J. Vlieghe y P. Zamojski, Manifesto for a Post-Critical Pedagogy (pp. 23-34). London: Punctum Books. Recuperado de: https://punctumbooks.com/titles/manifesto-for-a-postcritical-pedagogy/ (Consultado el 08/04/2020).

McLaren, P. (2012). La pedagogía crítica revolucionaria. El socialismo y los desafíos actuales. Buenos Aires: Herramienta.

Manifiesto latinoamericano de pedagogía crítica y educación popular (2015). Recuperado de: http://otrasvoceseneducacion.org/wp-content/uploads/2016/04/Manifiesto-Latinoamericano-de-Pedagog\%C3\%ADa-Cr\%C3\%ADtica-y-Educación-Popular.pdf (Consultado el 08/04/2020). 
Noguera-Ramírez, C. (2012). El gobierno pedagógico. Del arte de educar a las tradiciones pedagógicas. Bogotá: Siglo del Hombre Editores.

Noguera-Ramírez, C. y Marín-Díaz, D. (2015). Training the Human Animal: Biopolitics and Anthropotechnics. Sisyphus. Journal of education, 3(3), 110-131. Recuperado de: http:// revistas.rcaap.pt/sisyphus/article/view/8909/6408 (Consultado el 08/04/2020).

Senellart, M. (2006). As artes de governar. Do regimem medieval ao conceito de governo. São Paulo: Editora 34.

Sloterdijk, P. (2011). Reglas para el parque humano. En, Sin salvación. Tras las huellas de Heidegger. Madrid: Akal.

Sloterdijk, P. (2012). Has de cambiar tu vida. Valencia: Pre-Textos.

Silva, T. (1999). Documentos de identidade. Uma introdução às teorias do curriculo. Belo Horizonte: Autêntica. 